\title{
Soft Robotics. Bio-inspired Antagonistic Stiffening
}

\author{
Agostino Stilli ${ }^{1}$, Kaspar Althoefer ${ }^{2}$, and Helge A Wurdemann ${ }^{3}$ \\ 1 Department of Computer Science, University College London \\ London WC1E 7JE, UK \\ a.stilli@ucl.ac.uk \\ 2 School of Engineering and Materials Science \\ Queen Mary University of London, London E1 4NS, UK \\ 3 Department of Mechanical Engineering, University College London \\ London WC1E 7JE, UK \\ h. wurdemann@ucl.ac.uk, http://softhaptics.website
}

\begin{abstract}
Soft robotic structures might play a major role in the 4 th industrial revolution. Researchers have successfully demonstrated advantages of soft robotics over traditional robots made of rigid links and joints in several application areas including manufacturing, healthcare and surgical interventions. However, soft robots have limited ability to exert larger forces when it comes to interaction with the environment, hence, change their stiffness on demand over a wide range. Stiffnesscontrollability can be achieved as a result of the equilibrium of an active force and a passive reaction force or of two active forces antagonistically collaborating. In this paper, we present a novel design paradigm for a fabric-based Variable Stiffness System and its potential applications.
\end{abstract}

Keywords: bio-inspiration, stiffness-controllability, antagonistic actuation, soft robotics

\section{Introduction}

With the growing interest in the use of elastic materials for the creation of highly dexterous robots [1], material science has made inroads in the soft robotics community and become of paramount importance when creating soft robotic structures. A clear indication is the growth of publications about innovative soft material robots in recently appearing monothematic journals such as "Soft Robotics" [2] and dedicated sessions at major robotics conferences, e.g., ICRA and IROS. Some roboticists argue that soft robotic technologies will play a key role in the 4th industrial revolution [3] for safe human-robot interaction in manufacturing $[4,6]$, healthcare [7], and minimally invasive surgery (MIS) [8]. Numerous proposals for novel flexible robots based on soft and hybrid materials are continuously emerging [9]. Continuum hyper-redundant designs have been extensively investigated to create soft robots, for applications in surgery [10] with embedded sensors [11-13], disaster scenarios [14] and underwater exploration [15]. 
Although recent advances in soft and soft material robotics are notable and holding considerable promise to achieve what has not been possible with traditional rigid-linked robots, one important drawback remains: Despite their morphological capabilities, they have limited ability to exert larger forces on the environment when required, hence, change their stiffness on demand over a wide range [16]. In the search for the right trade-off between desired compliance and exertable force, researchers explored numerous approaches to enable on-demand stiffness tuning of soft robots. According to the recent comparative study presented in [16], Variable Stiffness Systems (VSSs) for soft robots can be divided in two main groups: (i) Active VSSs (AVSSs): these VSSs provide on-demand stiffening using an antagonistic approach, i.e., the creation of stiffness by means of equilibrium between two or more forces, at least one of which is an active force and (ii) Semi-Active VSSs (SAVSSs): these VSSs provide on demand stiffening relying on their capability of intrinsically tuning the rigidity of the robotic system in which they are embedded. In particular, recent works in [17-20] and [21] open up new avenues in this area by proposing inflatable robotic devices for applications in difficult-to-access sites, as in MIS and remote inspection. These devices can be highly compacted when in their undeployed, folded state and can be expanded in volume to multiples of the folded-state volume by injecting fluid and changing its stiffness by multiple times.

Our paper presents Variable Stiffness Systems based on a novel design paradigm for fabric-based soft robots. These stiffness mechanisms are inspired by nature and based on an active and passive antagonistic actuation principle. Here, we described the generic design, fabrication process and capabilities of these robotic systems including potential application areas that have been explored.

\section{Bio-inspired embodiment of a stiffness mechanism}

The proposed stiffness mechanism in this paper is inspired by biology: The role model for our research is the arm of the octopus. The octopus arms are made of longitudinal and transversal muscles [22]. Activating these muscle pairs that are distributed along the arms, the octopus is able to achieve high stiffness values. In other words, the sets of muscles "collaborate" in an opposing way to antagonistically stiffen the entire arm or arm segments. Hence, the octopus' arms are muscular hydrostats and can alternate between soft and rigid states combining advantages associated with both soft and hard systems by selectively controlling the stiffness of various parts of the body depending on the task requirements. In octopus arms, scientists have identified connecting tissue that keeps the muscles of the octopus arms in place, avoiding bulging and allowing the animal to achieve stiffness in their arms (comparable to a tube inside a bicycle tire). Our proposed actuation approach is antagonistic in its nature as it is the case in the above animal as well as many other animal and humans. To achieve similar behavior, we have here combined compliant (silicone and rubber materials) and non-compliant (fabric meshes and textiles) materials with the passive and active antagonistic manipulation principles. 


\section{The Variable Stiffness Link: A novel active structural element}

Silicone-based structures have been widely explored which aim at imitating biological behavior and achieving robotic solutions for complex challenges. The morphology of these robotic structures has been exploited creating adaptable systems capable of inherently safe human- and environment-robot interaction. The main idea is here to fabricate chambers of complex shapes with embedded fluidic actuators creating active variable stiffness systems, both, in active-active and active-passive configurations. To achieve a wide range of force and stiffness variation, braided material has been integrated to provide additional structural constraints to the chambers, thus, limiting undesired deformations - also known as ballooning. However, spacing between threads forming the braiding occurs and increases at high pressure values resulting in limitations.

\subsection{Design paradigm and methodology}

To prevent the aforementioned ballooning phenomena, we propose a novel design paradigm: the use of fabric as external braiding for fluidically actuated soft robotic structures. This approach has been firstly implemented in the creation of a novel active structural element called the Variable Stiffness Link (VSL). The proposed system is shown in Figure 1 (a) and comprises a hollow cylindrical structure made of silicone material, an embedded plastic mesh and an external nylon fabric sleeve. The internal airtight cylindrical chamber is supplied with pressurized air controlling the stiffness of the system. On the one hand, the plastic mesh guarantees shape retention at low pressures, on the other hand, the fabric layer provides a robust shape constraint at high pressures preventing any undesired deformation. The balance between the internal air pressure and the

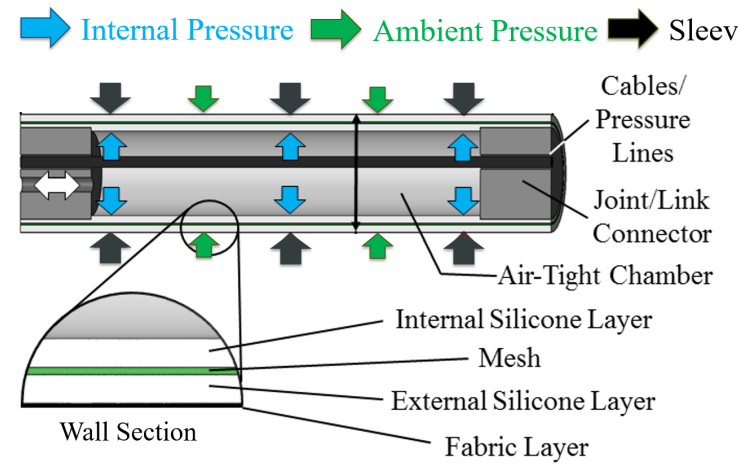

(a)

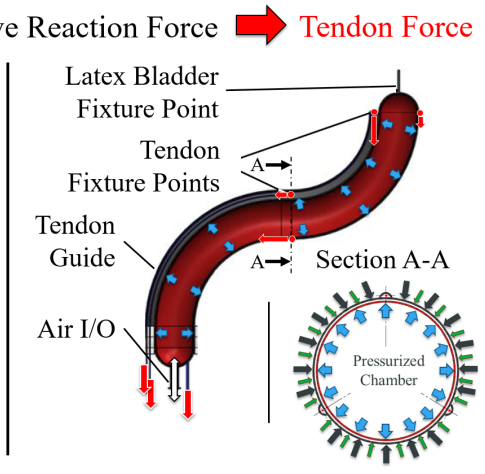

(b)

Fig. 1. Cross-sectional view of (a) the Variable Stiffness Link made of a plastic mesh embedded into silicone material and (b) the Inflatable Arm made of a latex bladder inside a fabric sleeve. 
reaction force by the fabric sleeve defines the stiffness of the VSL. Interfacing the VSL with a pressure regulator which monitors the current internal pressure allows to detect rapid changes in pressure values resulting from physical interaction with the environment.

\subsection{Materials and Fabrication}

The fabrication process of the VSL is as follows: a rectangular sheet of polypropylene diamond-shaped mesh is closed in a cylindrical shape by sealing the two overlapping edges at low temperature. The overlap is kept to a minimum in order to minimize the thickness increase after sealing keeping the system isomorphic. By using a commercially available heat sealer, a sealing line is produced on the rolled-up rectangular mesh forming the mesh into the shape of a cylinder. During the second stage of the fabrication process, the plastic mesh is embedded into a layer of silicone. A two-phase molding process is applied to cast silicone material on the mesh into a cylindrical shape. The result is a light-weight cylindrical-shaped stiffness-controllable element with a large internal lumen.

\section{Bio-inspired actuation for a soft continuum manipulator}

Built on the pneumatic actuation of the VSL, we have further explored robotic structures that are able to change its stiffness as well as shape. To enable shape shifting and shape locking capabilities, an additional actuation means has been introduced. Taking inspiration from the stiffening mechanism of natural muscles, we have developed a novel design that makes use of the extension behavior of pneumatic actuators and the contraction behavior of tendon-driven actuators resulting in an active variable stiffness system with active-active configuration. This concept has been firstly implemented in a continuum robotic manipulator called the Inflatable Arm.

The actuation principle and the design of the Inflatable Arm are illustrated in Figure 1 (b). The proposed system comprises three main elements: an internal airtight, yet expandable, latex bladder; an external, non-expandable, but collapsible and foldable polyester sleeve; and nylon tendons that are mounted to the outer fabric sleeve. Three tendons are fixed at the manipulator's tip and another set of three is attached to the outer fabric halfway between base and tip, $120^{\circ}$ spaced apart along the perimeter of the outer sleeve. The pushing force of the pressurized air inflates the manipulator and provides a straitening momentum, while the pulling force of the tendons steer the manipulator in the desired direction. A stable equilibrium between these forces can be achieved in any configuration providing the desired stiffness-controllability, shape-shifting and shape-locking capabilities. To show the potential of this approach in different application areas, this design paradigm has been implemented in two robotic systems, the Inflatable Endoscope and the Inflatable Exoskeleton Glove (INFLEXOGlove). 


\section{Potential applications for industrial settings and healthcare}

Three systems have been created to demonstrate the successful application of the presented robotic structures with embedded bio-inspired antagonistic stiffening:

- Figure 2 (a) illustrates the concept for a collaborative robot made of VSLs. The idea is to replace the rigid links of serial robots with VSLs. Hence, it is possible to change the stiffness of the links by varying the value of pressure inside their structure. Moreover, pressure readings from the pressure sensors inside the regulators can be utilized to detect collisions between the manipulator body and a human worker, for instance.

- Figure 2 (b) shows an inflatable, stiffness-controllable endoscope for minimally invasive surgery. Due to the nature of the used outer material and its soft, compressible structure, the proposed device is inherently safe when physically interacting with soft tissue.

- Figure 2 (c) proposes a light-weight inflatable soft exoskeleton device, called the InflExoGlove, to deliver gradual rehabilitation therapy in order to deliver effective high-dosage rehabilitation therapies for post-stroke disabilities.

\section{References}

1. Bauer, S., Bauer-Gogonea, S., Graz, I., Kaltenbrunner, M., Keplinger, C., Schwoediauer, R.: 25th anniversary article: a soft future: from robots and sensor skin to energy harvesters. Adv. Mater. 26(1), 149162 (2014)

2. Robinson, G., Davies, J.B.C.: Continuum robots - a state of the art. IEEE Int. Conf. Robot. Autom. 4 (1999)

3. Rossiter, J., Hauser, H.: Soft robotics the next industrial revolution. IEEE Robot. Autom. Mag. 23, 17-20 (2016)

4. Stilli, A., Wurdemann, H.A., Althoefer, K.: A Novel Concept for Safe, StiffnessControllable Robot Links. Soft Robot. 4(1), 16-22 (2016)

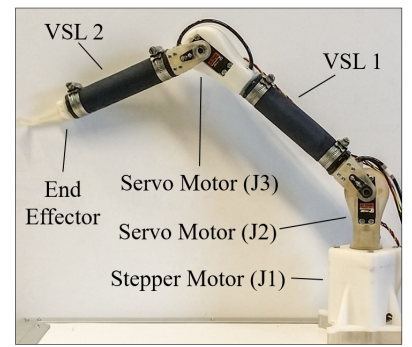

(a)

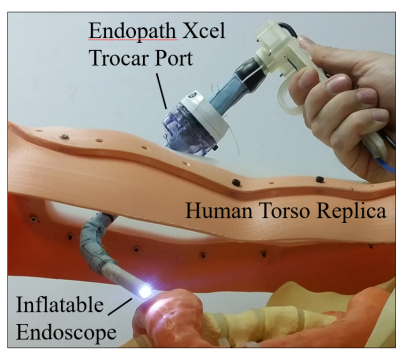

(b)

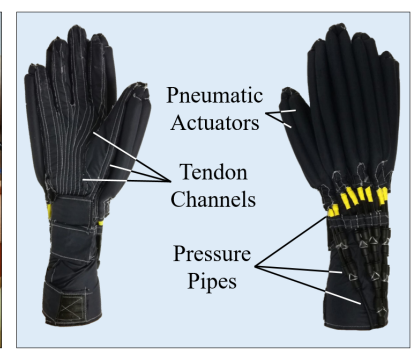

(c)

Fig. 2. Applications of the presented novel design paradigm: (a) a cobot made of Variable Stiffness Links, (b) an inflatable and stiffness-controllable endoscope and (c) the INFLatable EXOskeleton Glove (InflExoGlove). 
5. Stilli, A., Grattarola, L., Feldmann, H., Wurdemann, H.A., Althoefer, K.: Variable Stiffness Links VSL - Toward Inherently Safe Robotic Manipulators. IEEE Int. Conf. Robot. Autom., 4971-4976 (2017)

6. Pfeifer, R., Marques, H.G., Iida, F.: Soft robotics: the next generation of intelligent machines. Int. joint Conf. on Artificial Intelligence, 5-11 (2013)

7. Horvath, M.A., Wamala, I., Rytkin, E., Doyle, E., Payne, C.J., Thalhofer, T., Berra, I., Solovyeva, A., Saeed, M., Hendren, S., Roche, E.T., Pedro, J., Walsh, C.J., Vasilyev, N.V.: An Intracardiac Soft Robotic Device for Augmentation of Blood Ejection from the Failing Right Ventricle. Ann. Biomed. Eng. 45(9), 2222-2233 (2017)

8. Arezzo, A., Mintz, Y., Allaix, M.E., Arolfo, S., Bonino, M., Gerboni, G., Brancadoro, M., Cianchetti, M., Menciassi, A., Wurdemann, H.A., Noh, Y., Althoefer, K., Fras, J., Glowka, J., Nawrat, Z., Cassidy, G., Walker, R., Morino, M.: Total mesorectal excision using a soft and flexible robotic arm: a feasibility study in cadaver models. Surg. Endosc. 31(1), 264273 (2016)

9. Lipson, H.: Challenges and opportunities for design, simulation, and fabrication of soft robots. Soft Robot. 1(1), 21-27 (2014)

10. Burgner-Kahrs, J., Rucker, D.C., Choset, H.: Continuum robots for medical applications: A survey. IEEE Trans. Robot. 31(6), 1261-1280 (2015)

11. Faragasso, A., Stilli, A., Bimbo, J., Noh, Y., Liu, H., Nanayakkara, T., Dasgupta, P., Wurdemann, H.A., Althoefer, K.: Endoscopic add-on stiffness probe for real-time soft surface characterisation in MIS. Int. Conf. of the IEEE Engineering in Medicine and Biology Society, 6517-6520 (2014)

12. Sareh, S., Jiang, A., Faragasso, A., Nanayakkara, T., Dasgupta, P., Seneviratne, L., Wurdemann, H., Althoefer, K.: MR-Compatible Bio-Inspired Tactile Sensor Sleeve for Surgical Soft Manipulators. IEEE Int. Conf. Robot. Autom., 1454-1459 (2014)

13. Wurdemann, H.A, Sareh, S., Shafti, A., Noh, Y., Faragasso, A., Chathuranga, D.S., Liu, H., Hirai, S., AlthoeferK.: Embedded electro-conductive yarn for shape sensing of soft robotic manipulators. Int. Conf. of the IEEE Engineering in Medicine and Biology Society, 8026-8029 (2015)

14. Kamegawa, T., Yarnasaki, T., Igarashi, H., Matsuno, F.: Development of the snakelike rescue robot kohga. IEEE Int. Conf. Robot. Autom., 5081-5086 (2004)

15. Crespi, A., Badertscher, A., Guignard, A., Ijspeert, A.J.: AmphiBot I: an amphibious snake-like robot. Rob. Auton. Syst. 50(4), 163-175 (2005)

16. Manti, M., Cacucciolo, V., Cianchetti, M.: Stiffening in soft robotics: A review of the state of the art. IEEE Robot. Autom. Mag. 23(3), 93-106 (2016)

17. Stilli, A., Wurdemann, H.A., Althoefer, K.: Shrinkable, stiffness-controllable soft manipulator based on a bio-inspired antagonistic actuation principle. IEEE Int. Conf. on Int. Robots and Syst., 2476-2481 (2014)

18. Maghooa, F., Stilli, A., Noh, Y., Althoefer, K., Wurdemann, H.A.: Tendon and pressure actuation for a bio-inspired manipulator based on an antagonistic principle. IEEE Int. Conf. Robot. Autom., 2556-2561 (2015)

19. Wurdemann, H., Stilli, A., Althoefer, K.: An antagonistic actuation technique for simultaneous stiffness and position control. Int. Robot. and Apps., 164-174 (2015)

20. Shiva, A., Stilli, A., Noh, Y., Faragasso, A., Althoefer K., Wurdemann, H.A.: Tendon-based stiffening for a pneumatically actuated soft manipulator. IEEE Robot. Autom. Lett. 1(2), 632-637 (2016)

21. Hawkes, E.W., Blumenschein, L.H., Greer, J.D., Okamura, A.M.: A soft robot that navigates its environment through growth. Sci. Robot. 2(8) (2017)

22. Cianchetti, M., Arienti, A., Follador, M., Mazzolai, B., Dario, P., Laschi, C.: Design concept and validation of a robotic arm inspired by the octopus, Materials Science and Engineering C 31, 1230-1239 (2011) 